\title{
Methods of accurate localisation of non-palpable subdermal contraceptive implants
}

\author{
Diana Mansour, lan S Fraser, Martyn Walling, Derek Glenn, Olivier Graesslin, Christian Egarter, \\ Josef Herbst
}

\section{Introduction}

Implanon ${ }^{\circledR}$ (Organon International, Oss, The Netherlands) is a single rod, subdermal contraceptive implant system designed to release the progestogen etonogestrel at a near constant rate over 3 years. 1,2 This system entered the market in The Netherlands in 1998, and soon after in many other countries in Europe and Asia. It is now marketed in over 35 countries $^{3}$ and more than 3.3 million implants have now been sold worldwide. ${ }^{3}$ Many women have found Implanon to be a highly successful and convenient contraceptive, with more than one-third of users choosing a further implant at the end of the 3 -year period. ${ }^{4}$ The success of the Implanon system has followed on from the extensive experience gained in many centres worldwide with the first subdermal contraceptive implants, namely the levonorgestrel-releasing implant system, Norplant ${ }^{\circledR}$ (developed by the Population Council, New York and Leiras, Finland).

The clinical experience associated with this large number of insertions has inevitably been followed by extensive experience with implant removals. Many of these removals have occurred at around the end of the scheduled 3-year lifespan, but a large minority have occurred on patient request at an earlier date..$^{5}$ In most countries, training for removals has been an integral part of the Implanon training programme that is recommended for doctors who intend to offer Implanon to their patients. These programmes have all strongly emphasised the need for precise attention to the directions for correct insertion in order to ensure straightforward removal at a later date.

Since Implanon's launch 8 years ago, clinical

J Fam Plann Reprod Health Care 2008; 34(1): 9-12

(Accepted 18 November 2007)

Contraception and Sexual Health Service, Newcastle upon Tyne Primary Care Trust, Newcastle upon Tyne, UK

Diana Mansour, FRCOG, FFFP, Consultant in Community Gynaecology

Department of Obstetrics and Gynaecology, University of Sydney, Sydney, Australia

Ian S Fraser MD, FRANZCOG, Professor in Reproductive Medicine

Parkside Surgery, Boston, UK

Martyn Walling, FRCGP, FFFP, General Practitioner

Department of Radiology, St George Hospital, Sydney,

Australia

Derek Glenn, MBBS, FRANZCR, Director of Radiology

Department of Obstetrics and Gynaecology, Centre

Hospitalier Universitaire, Reims, France

Olivier Graesslin, MD, Gynaecologist

Department of Obstetrics and Gynaecology, University of Vienna Medical School, Vienna, Austria

Christian Egarter, MD, Professor

Department of Obstetrics and Gynaecology, Paracelsus Klinik, Marl, Germany

Josef Herbst, MD, Head of Department

Correspondence to: Dr Diana Mansour, Contraception and Sexual Health Service, Newcastle upon Tyne Primary Care Trust, Graingerville Clinic, Westgate Road, Newcastle upon Tyne NE4 6BE, UK. E-mail: Diana.Mansour@newcastle-pct.nhs.uk experience has generally been very positive, with most practitioners having no difficulty in locating the implant requiring removal by palpation, and then following the simple instructions for safe and expeditious removal. It is estimated that only a very small proportion of Implanon implants are incorrectly inserted and that less than $0.1 \%$ result in difficult removals. ${ }^{3}$ However, accumulating experience in a small number of specialised centres is now beginning to define the issues facing the practitioner who encounters a patient requesting Implanon removal when the implant is not palpable. 6

\section{Implant insertion technique Norplant insertion}

Much of our insertion 'experience' worldwide has been initially based on inserting the six-capsule levonorgestrel contraceptive implant system, Norplant. ${ }^{7}$ These implants were inserted in the subdermal layer on the medial side of the non-dominant upper arm. ${ }^{7}$ Although no specific location was described, most clinicians inserted the implants over the anterior surface of the biceps.

In practice it was not uncommon to see some migration of Norplant capsules; however, if correctly placed, the extent of migration would not usually exceed $2 \mathrm{~cm} .8$ Clinicians also saw a few women who had requested that the implants be inserted in the thigh or abdomen. Inevitably these implants were far more difficult to locate and remove. The upper arm was chosen as the ideal site for placement of contraceptive implants as it is a closed anatomical compartment with extensive migration unlikely.

\section{Implanon insertion}

With the introduction of Implanon, the single, non-biodegradable implant rod measuring $40 \times 2 \mathrm{~mm}$, insertion and removal became easier. ${ }^{9}$ Implanon, unlike Norplant, is preloaded within a sterile, disposable applicator (Figure 1).

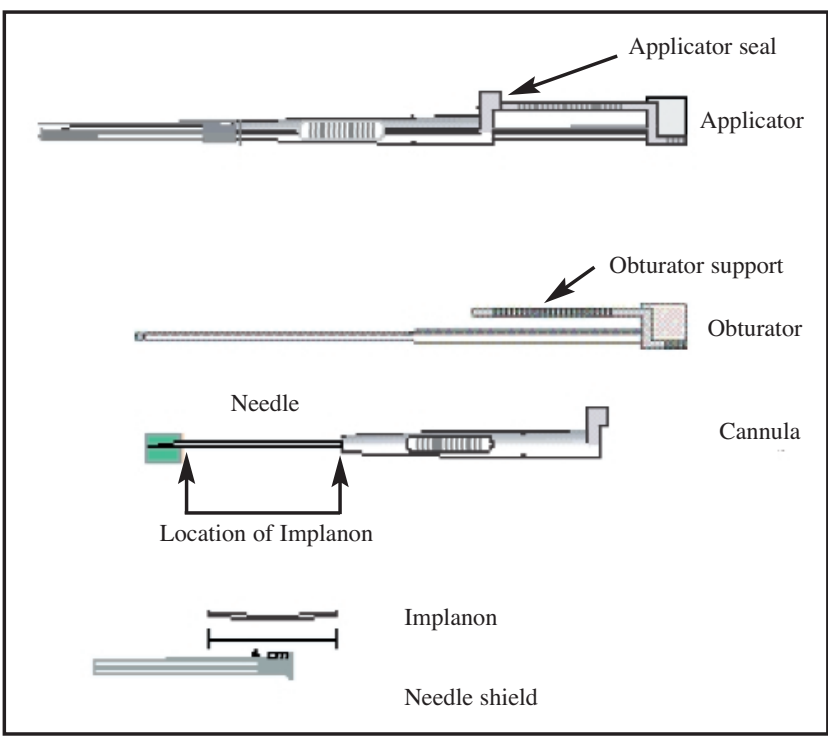

Figure 1 Diagrammatic representation of the Implanon ${ }^{\circledR}$ applicator. (C) Organon International. Figure reproduced with the kind permission of the copyright holder 


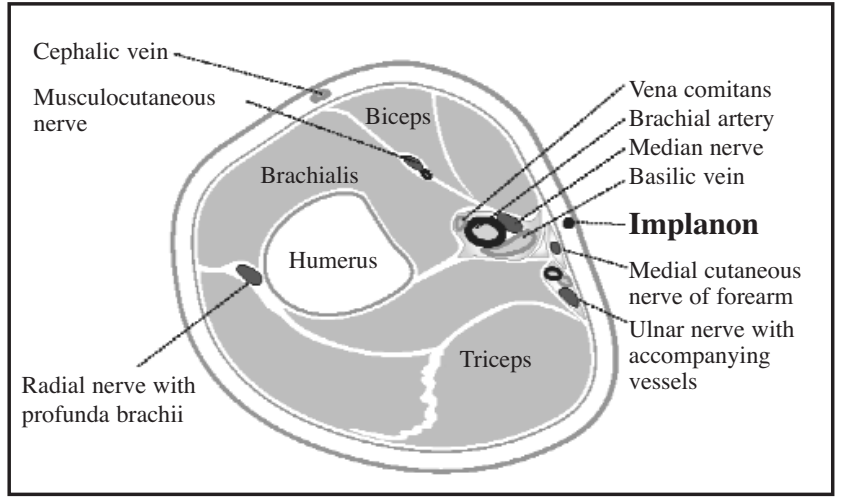

Figure 2 Transverse section through the left arm just below the mid-shaft of the humerus demonstrating the original recommended insertion location of Implanon ${ }^{\circledR}$. (C) Organon International. Figure reproduced with the kind permission of the copyright holder

The most recently approved manufacturer's Product Information states that Implanon should be inserted at the inner side of the non-dominant arm about 8-10 $\mathrm{cm}$ above the medial epicondyle. However, previous instructions provided by the manufacturer ${ }^{10}$ stated that the implant should be placed subdermally at the inner side of the nondominant upper arm about $7 \mathrm{~cm}$ above the elbow crease in the groove between the biceps and the triceps (Figure 2). Clinicians who had previous experience of inserting and removing Norplant, however, frequently continued to insert this single rod over the anterior border of the biceps. Hence there are minor variations in the site at which a 'lost' Implanon may have been originally inserted.

The procedure is carried out using an aseptic technique under local anaesthetic, which is injected along the intended insertion channel. The needle of the Implanon inserter is introduced under the skin, and by tenting the skin with the tip of the needle this is advanced in a cranial direction to its full length, entirely within the immediate subdermal layer. The obturator is turned $90^{\circ}$ and firmly secured in place. The needle is then slowly withdrawn over the obturator, releasing the implant into the correct plane and position in the arm. Following insertion, the distance between the implant and the needle entry point should be about $1 \mathrm{~cm}$. The implant is easily palpable if correctly positioned but is normally invisible. A slight depression in the overlying skin may be noticeable in a small number of women.

Studies have suggested that Implanon is approximately four times as quick to insert and remove when compared to the multi-implant levonorgestrel system, Norplant, and is associated with a significantly lower incidence of removal complications. ${ }^{11}$ Incorrect insertion makes removal much more difficult, a key point emphasising the importance of recommended training.

\section{Possible insertion errors and unusual anatomical sitings of non-palpable Implanon implants}

Most experts equate non-palpable Implanon rods with poor insertion technique. The procedure for insertion has been well described and trainers have been encouraged to emphasise the superficial subdermal placement of the implant, parallel to the skin. Prior to insertion the clinician should check that the implant is within the needle of the applicator, and following removal of the protective cap the needle must be kept upright. There have been anecdotal reports of implants falling onto the floor or trolley as the clinician picks up the applicator prior to insertion while holding the needle in a downward angle. This may result in a non-insertion and possible unplanned pregnancy. ${ }^{12}$
At insertion, the skin should be tented while the insertion needle is advanced parallel to the skin. Failure to follow these instructions may result in deep subcutaneous or intramuscular insertion. It is not uncommon to find the proximal end of the rod deeper than the distal end, implying that after insertion the point of the needle was directed at a steep angle into the arm rather than parallel to the $\operatorname{skin}^{13}$ (Figure 3). Thin women with very scant subcutaneous tissue may also pose a deep insertion risk with the biceps fascia being breached inadvertently by the very sharp insertion needle. Even though the implant is palpable, removal can be difficult since the muscle fascia must be incised. To prevent such problems arising during insertion in thin women, some experts advise using a moderate amount of local anaesthetic (4-5 $\mathrm{ml} 1 \%$ lignocaine) to separate the tissue planes, allowing easier subcutaneous implant placement.

The obturator must be stabilised when the needle is withdrawn, otherwise the implant may remain within the applicator as the whole system is withdrawn, resulting in a non-insertion. Conversely, firm forward pressure on the obturator during withdrawal of the needle may push the implant up towards the axilla.

Occasionally, women present with 'lost' Implanon implants and on closer inspection the contraceptive is found in the other arm. Rare reports also mention finding the implant on the lateral side of the upper arm, or in the thigh. Care must be taken when replacing old with new contraceptive implants, particularly when removing Norplant or Jadelle ${ }^{\circledR}$ and inserting Implanon through the same skin incision. The new single implant may be inadvertently placed more deeply in the subcutaneous tissue owing to tissue disruption and oedema caused by the multi-rod removal process.

There is little evidence that Implanon migrates more than $2 \mathrm{~cm}$ if correctly and superficially placed, even when it is over the biceps muscle. ${ }^{14}$ There have been reports from clinicians suggesting greater migration if the implant is deeply inserted intramuscularly. Other anecdotal evidence suggests that some women 'play' with their implant, pushing it up and down their arm or bending it. As the rod is semi-rigid this can result in additional migration and sometimes partial breakage of the implant.

Case reports from centres worldwide give similar results to published findings ${ }^{13}$ that approximately $70 \%$ of non-palpable implants are located deep in subcutaneous fat and the remainder under the muscle fascia within biceps or

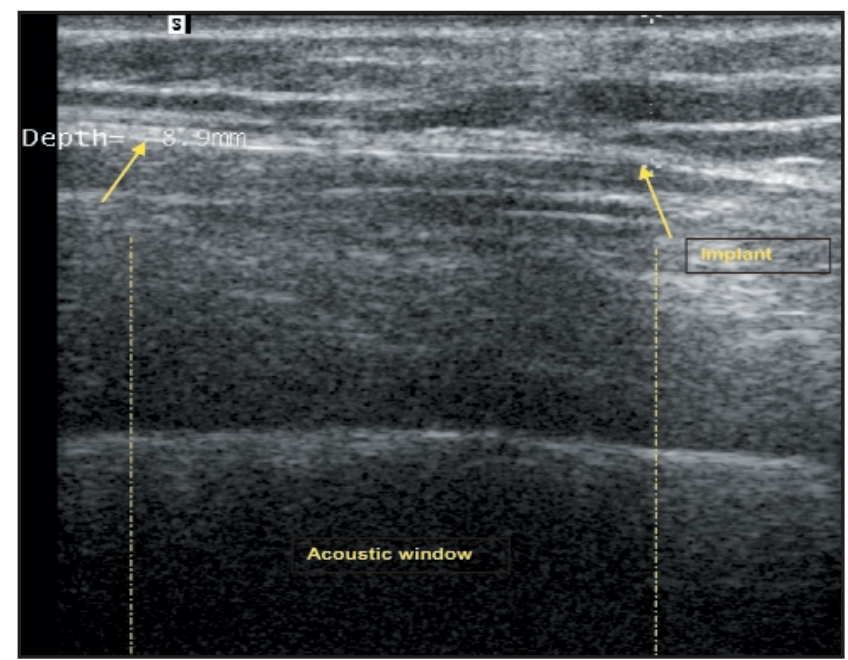

Figure 3 Deep location of Implanon ${ }^{\circledR}$ using ultrasound demonstrating the varying depths of the proximal and distal ends. ${ }^{13}$ Figure reproduced with the kind permission of D Mansour 


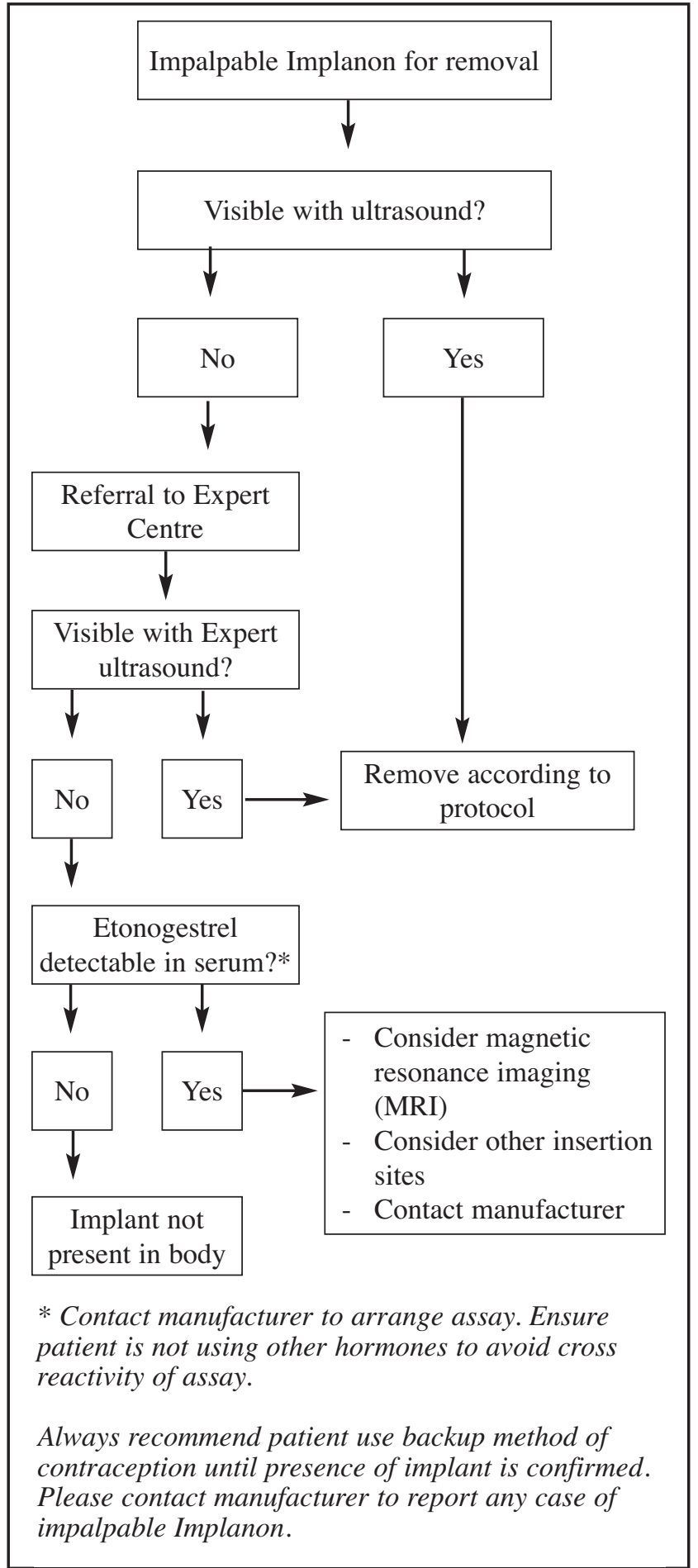

Figure 4 Flow chart illustrating the steps required for identifying 'lost' Implanon ${ }^{\circledR}$ implants. Figure prepared with the assistance of all seven authors

triceps. Implanon is rarely found within the neurovascular bundle. ${ }^{15}$

\section{Approach to localisation of non-palpable Implanon implants}

In cases of non-palpable implants it is worthwhile taking a careful history from the patient. Find out whether the implant has ever been felt and, if so, when it 'disappeared'. Was there prolonged discomfort or pain in the arm after insertion, which may indicate deep location of the implant? Have there been any untoward recent events such as pregnancy, indicating non-insertion of Implanon?
It is helpful to ask the patient to demonstrate the position of the arm when the implant was inserted and, if possible, to show you the insertion scar. Both arms should be examined from the elbow to the axilla to look for possible 'insertion' marks. These are often not obvious but correct identification will aid implant location by ultrasound. The arms should be gently palpated using fingertips to help identify deep but palpable implants. Be aware of anatomical structures such as muscle borders and neurovascular bundles in the higher regions of the inner side of the upper arm, which have to be distinguished from an implant by conducting palpation with sufficient care for detail. If there is any doubt, follow the algorithm shown in Figure 4 to help locate 'lost' Implanon implants.

\section{Ultrasound for localisation}

Most clinicians with experience in locating deeply placed implants recommend ultrasound as the investigation of choice. The arm is placed in the implant 'insertion' position and coupling gel applied to the upper arm. The transducer is positioned transversely (perpendicular to the length of the humerus) in the region of the insertion scar. As early as 1997, key sonographic features were described for identifying these implants including the small echogenic 'dot' of the Implanon and, most importantly, the posterior acoustic shadowing seen when the arm is scanned transversely (Figure 5). ${ }^{16}$

The anatomical position of the implant is noted (e.g. within subcutaneous tissue or deep to muscle fascia) and its depth from the skin surface measured. The proximal and distal ends of the implant are located by scanning transversely along the arm and the skin marked to assist removal.

Inexpensive portable machines can be used as long as they have a high resolution linear array transducer (7-14 $\mathrm{MHz}$, preferably $12-14 \mathrm{MHz}) .{ }^{17}$ If more complex equipment is employed then fundamental rather than compound imaging is advised and image processing software such as SonoCT ${ }^{\circledR}$ (Philips, Reigate, UK) or similar should be disabled. Advanced ultrasound technology automatically reduces 'shadowing' to improve image quality. ${ }^{18}$ This shadowing, however, is the key to successful location of impalpable implants. A single rather than multiple focal points should be selected and this single focus should be set at about $1 \mathrm{~cm}$ below the skin.

If the implant cannot be successfully located by a doctor experienced with ultrasound localisation of Implanon then the manufacturer should be notified. They will arrange for serum etonogestrel levels to be performed at their laboratory in The Netherlands. As some exogenous progestogens crossreact with this assay, women must be advised to use non-hormonal contraception until blood has been taken. In most cases clinicians only want to know if etonogestrel is present in the body, rather than an accurate serum level, so the serum sample can be transported at room temperature. If a quantitative test is required then the serum must be transported 'on ice'. If no etonogestrel is detected in the assay then the Implanon is no longer in the body. Theoretically, the Implanon may have been in the body so long that etonogestrel levels are undetectable, but this has not yet been recorded (even with implants left in place for up to 7 years).

If ultrasound by an experienced clinician, familiar with the characteristic acoustic signature of an Implanon, using appropriate equipment and scanning protocols fails to locate the non-palpable implant the manufacturer advises using magnetic resonance imaging (MRI) technology. MRI, however, is costly and caution is required when differentiating between blood vessels, fibrous septae and 


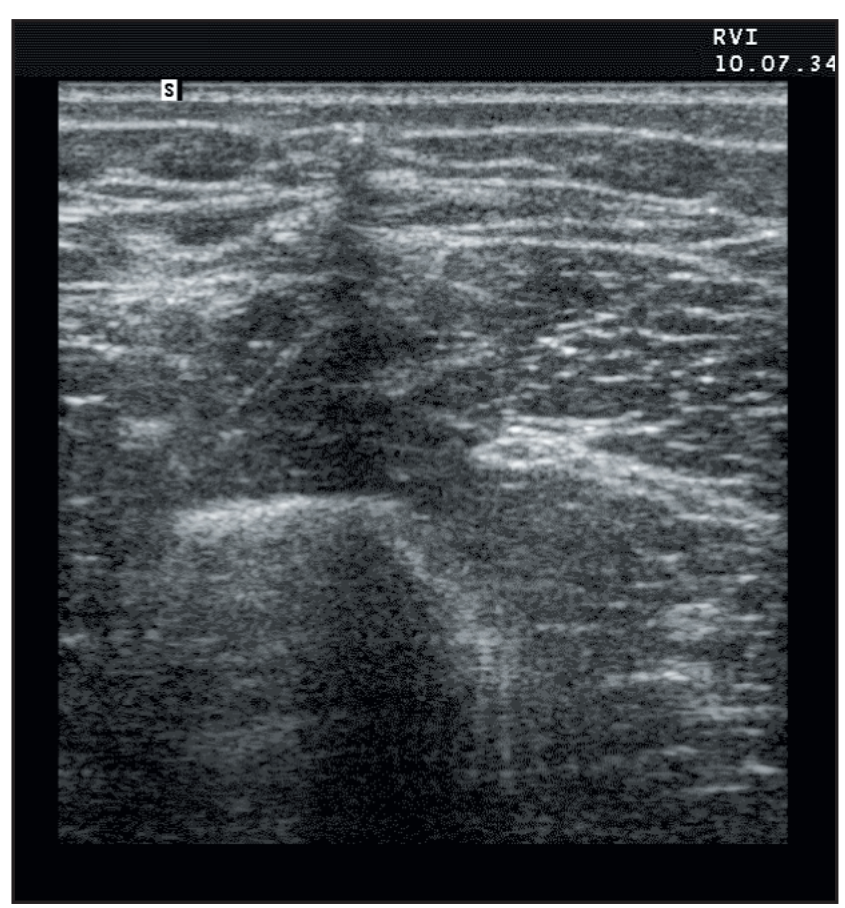

Figure 5 Ultrasound scan locating a deeply placed Implanon ${ }^{\circledast}$ implant (below the muscle fascia) in the transverse plane. ${ }^{13}$ Figure reproduced with the kind permission of D Mansour

Implanon rods. ${ }^{19,20}$ Even if MRI is used to determine that an implant is present, subsequent ultrasound is still required to precisely determine the implant position at the time of removal.

\section{New developments}

The manufacturer of Implanon is currently working on a radio-opaque implant, which may make projection radiography a useful addition to the imaging approaches currently used for localisation of non-palpable implants. A newly designed applicator preloaded with the radio-opaque implant will aid correct superficial insertion and precise subdermal location of the implant.

\section{Conclusions}

The vast majority of subdermal contraceptive implants are correctly inserted and are easily palpable at the time of planned removal. This means that very few practitioners gain more than occasional experience of the removal of non-palpable implants. The key to safe and effective removal is initial accurate localisation using the correct ultrasound probe, with recognition of the typical signal generated by the implant. The implant can then be safely removed using an appropriate and recognised technique. ${ }^{13,15}$

Inexperienced operators should not hesitate to contact an experienced centre or Organon International prior to embarking on a difficult removal.
Acknowledgements

The authors are grateful to Maaike Addicks at Organon International for advice on proprietary matters and for assistance with illustrations.

Statements on funding and competing interests

Funding All the authors have received honoraria and expenses for attendance at advisory boards, lectures or sponsored symposia for Organon.

Competing interests None identified.

References

1 Darney PD. Implantable contraception. Eur J Contracept Reprod Health Care 2000; 5(Suppl. 2): 2-11.

2 Wenzl R, van Beek A, Schnabel P, Huber J. Pharmacokinetics of etonogestrel released from the contraceptive implant Implanon. Contraception 1998; 58: 283-288.

3 Organon International. Data on file.

4 Lakha F, Glasier AF. Continuation rates of Implanon in the UK: data from an observational study in a clinical setting. Contraception 2006; 74: 287-289.

5 Flores JB, Balderas ML, Bonilla MC, Vazquez-Estrada L. Clinical experience and acceptability of the etonogestrel subdermal contraceptive implant. Int J Gynaecol Obstet 2005; 90: 228-233.

6 Fraser IS. The challenges of location and removal of Implanon contraceptive implants. J Fam Plann Reprod Health Care 2006 32: $151-152$.

7 Fraser IS, Tiitinen A, Affandi B, Brache V, Croxatto HB, Diaz S, et al. Norplant consensus statement and background review. Contraception 1998; 57: 1-9.

8 Oloto EJ, Bromham DR. Norplant removal: a review. Br J Fam Plann 1995; 21(Suppl.): 21-24.

9 Edwards JE, Moore A. Implanon - a review of clinical studies. Br J Fam Plann 1999; 4(Suppl): 3-16.

10 Electronic Medicines Compendium. Implanon $68 \mathrm{mg}$ implant for subdermal use (Summary of Product Characteristics). http://emc.medicines.org.uk/emc/assets/c/html/DisplayDoc.asp ?DocumentID=5382 [Accessed 4 November 2007].

11 Mascarenhas L. Insertion and removal of Implanon. Contraception 1998; 58: 79S-83S.

12 Harrison-Woolrych M, Hill R. Unintended pregnancies with the etonogestrel implant (Implanon): a case series from postmarketing experience in Australia. Contraception 2005; 71: 306-308.

13 Singh M, Mansour D, Richardson D. Location and removal of non-palpable Implanon ${ }^{\circledR}$ implants with the aid of ultrasound guidance. J Fam Plann Reprod Health Care 2006; 32: 153-156.

14 Ismail H, Mansour D, Singh M. Migration of Implanon ${ }^{\circledR}$. J Fam Plann Reprod Health Care 2006; 32: 157-159.

15 James P, Trenery J. Ultrasound localisation and removal of non-palpable Implanon implants. Aust N Z J Obstet Gynaecol 2006; 46: 225-228.

16 Lantz A, Nosher JL, Pasquale S, Siegel RL. Ultrasound characteristics of subdermally implanted Implanon contraceptive rods. Contraception 1997; 56: 323-327.

17 Walling M. How to remove impalpable Implanon ${ }^{\circledR}$ implants. $J$ Fam Plann Reprod Health Care 2005; 31: 320-321.

18 Gabriel H, Shulman L, Marko J, Nikolaidis P, Chirita V. Compound versus fundamental imaging in the detection of subdermal contraceptive implants. J Ultrasound Med 2007; 26: 355-359 (erratum in J Ultrasound Med 2007; 26: 1452).

19 Westerway SC, Picker R, Christie J. Implanon implant detection with ultrasound and magnetic resonance imaging. Aust N Z J Obstet Gynaecol 2003; 43: 346-350.

20 Shulman LP, Gabriel H. Management and localization strategies for the nonpalpable Implanon rod. Contraception 2006; 73: 325-330. The ideal reference for anyone working in this sector

Order on our secure website www.KeywaysPublishing.com or contact: Keyways Publishing, PO Box 100, Chichester, West Sussex, PO18 8HD, UK tel: 01243576444 fax: 01243576456 email: info@KeywaysPublishing.com 